\title{
Dinámica poblacional de tallos de ovillo (Dactylis glomerata L.) solo y asociado con ballico perenne (Lolium perenne L.) y trébol blanco (Trifolium repens L.)
}

\author{
Tiller population in orchard grass (Dactylis glomerata L.) \\ grown alone and associated with perennial ryegrass (Lolium \\ perenne L.) and white clover (Trifolium repens $\mathrm{L}_{\text {.) }}$
}

\author{
Adelaido Rafael Rojas Garcíaa, Joel Ventura Ríosa , Alfonso Hernández-Garaya*, Santiago Joaquín Cancinob, \\ María de los Ángeles Maldonado Peraltac, Iván Reyes Vázquez ${ }^{a}$
}

\begin{abstract}
RESUMEN
El objetivo de esta investigación fue evaluar la densidad de tallos del pasto ovillo en monocultivo, y asociado con ballico perenne y trébol blanco en diferentes proporciones. Los tratamientos consistieron de las siguientes asociaciones: 20-70-10, 70-20$10,100-00-00,40-40-20 \%$ de pasto ovillo $(\mathrm{Ov})$, ballico perenne (Ba) y trébol blanco (Tr), respectivamente. Los tratamientos se distribuyeron en un arreglo de bloques al azar con tres repeticiones. La asociación 70-20-10 de Ov-Ba-Tr es la que presenta mayor densidad de tallos de $\mathrm{Ov}$, con un promedio de 3,750 tallos $\mathrm{m}^{-2}$, y la menor densidad de tallos fue en el Ov en monocultivo (100-00$00)$, con un promedio de 2,400 tallos $\mathrm{m}^{-2}(P<0.05)$. La población de tallos en Ba fue baja en todas las asociaciones, en promedio de 225 tallos $\mathrm{m}^{-2}$. La mayor densidad de tallos en pasto Ov-Ba se presentó en otoño e invierno. Independientemente de la asociación en la estación de primavera se encontró mayor peso por tallo con 0.30 y $0.42 \mathrm{~g}$ tallo $^{-1}$, en $0 v$ y Ba y menor en invierno con 0.13 y $0.14 \mathrm{~g} \mathrm{tallo}^{-1}$, en Ov y Ba, respectivamente. En conclusión, la dinámica de población de tallos de pasto ovillo tendió a mantenerse; en contraste, en pasto ovillo solo y ballico perenne disminuyó la población de tallos.
\end{abstract}

PALABRAS CLAVE: Población de tallos, Peso de tallos, Otoño, Invierno.

\begin{abstract}
The objective of this research was to evaluate tiller population of orchard grass seeded with perennial ryegrass and white clover in different proportions and single. Treatments consisted of the associations: 20-70-10, 70-20-10, 100-00-00, 40-40-20 \% of orchard grass (OG), perennial ryegrass (RG) and white clover (WT) respectively. Treatments were distributed in a randomized complete block with three replications. The association 70-20-10 of OG-RG-WT had greatest tiller density of OG with an average of 3,750 tiller $\mathrm{m}^{-2}$, and the lowest in 100-00-00 with an average of 2,400 tiller $\mathrm{m}^{-2}(P<0.05)$. There were few tiller in perennial ryegrass in all associations, with an average of 225 tiller $\mathbf{m}^{-2}$. Two highest peaks in the tiller population in OG and RG were found in autumn and winter. Regardless of the association, in the spring season more weight per tiller was found with 0.30 and $0.42 \mathrm{~g}$ tiller-1, $0 \mathrm{G}$ and RG and less in winter with 0.13 and $0.14 \mathrm{~g} \mathrm{tiller}^{-1}, \mathrm{OG}$ and RG, respectively. In conclusion, all associations in population dynamics of OG tiller tended to be maintained; in contrast, on single OG and RG tended to decrease.
\end{abstract}

KEY WORDS: Tiller population, Tiller weight, Fall, Winter.

Recibido el 5 de abril de 2016. Aceptado el 24 de junio de 2016.

a Recursos Genéticos y Productividad Ganadería. Campus Montecillo. Colegio de Postgraduados. Km. 36.5 Carretera México - Texcoco. México.

b División de Estudios de Posgrado, Facultad de Ingeniería y Ciencias, Universidad Autónoma de Tamaulipas. Ciudad Victoria, Tamaulipas. México.

c Recursos Genéticos y Productividad Fisiología Vegetal. Campus Montecillo. Colegio de Postgraduados. Texcoco. México.

*Autor de correspondencia: hernan@colpos.mx 
En gramíneas o Poaceas, la unidad básica de crecimiento es el tallo, en su conjunto, por unidad de superficie forman una población, por lo tanto una pradera puede ser vista como una población de tallos ${ }^{(1)}$. Un aumento en la población de tallos significa mayor persistencia y producción de forraje $^{(2)}$. En una pradera, la densidad de tallos por unidad de superficie es continuamente variable según las condiciones ambientales, estación del año y manejo ${ }^{(3-7)}$. La producción de forraje en una pradera puede ser dividida en dos componentes: el número de tallos por unidad de área y el peso individual por tallo(4). Por ende, la persistencia y producción de las especies forrajeras depende del balance entre la formación y protección de nuevos tallos.

El conocimiento de la densidad y peso de los tallos proporciona una referencia esencial para el manejo de praderas. La intensidad y frecuencia de pastoreo, modifican la tasa de aparición y muerte de tallos $y$, con ello, incrementan la densidad y productividad de la pradera ${ }^{(3,8)}$. Diferentes investigadores, al evaluar praderas mixtas de leguminosas y gramíneas y en monocultivo, obtuvieron mayor compensación tamaño/densidad de tallos en las asociaciones, manteniéndose constante durante el año en comparación con praderas puras o de una especie sola ${ }^{(9)}$. En praderas de ballico perenne (Lolium perenne L.) se reportó la mayor densidad de tallos en la estación de invierno y verano independientemente de la frecuencia de pastoreo ${ }^{(10)}$. En asociaciones de gramíneas y leguminosas registraron el mayor peso por tallo de ballico perenne y pasto ovillo (Dactylis glomerata L.) en verano (0.38 $\mathrm{g}$ tallo $^{-1}$ ) y la mayor densidad de tallos en invierno con 9,961 y 10,423 tallos $\mathrm{m}^{-2}$, respectivamente ${ }^{(11)}$. Otros investigadores ${ }^{(12)}$ reportaron la mayor densidad de tallos en pasto ovillo en invierno con 8,000 tallos $\mathrm{m}^{-}$ 2 disminuyendo en primavera, manteniéndose constante hasta verano con un una densidad de 4,421 tallos $\mathrm{m}^{-2}$ y aumentando en otoño.

En México existen pocos estudios sobre dinámica poblacional de tallos y componentes del rendimiento en asociaciones de gramíneas con leguminosas. Evaluar dichos componentes y su variación durante el año, genera información importante para la toma de decisiones que favorezcan simultáneamente una alta producción, utilización y persistencia de las especies forrajeras ${ }^{(13)}$. La presente investigación tuvo como objetivo evaluar las tasas de aparición, muerte, sobrevivencia y dinámica poblacional de tallos, así como el peso por tallo de ovillo y ballico perenne asociados con trébol blanco, y el ovillo en monocultivo.

El experimento se realizó de septiembre de 2012 a septiembre de 2014, en el Campo Experimental del Colegio de Postgraduados, Campus Montecillo, Texcoco, Estado de México, ubicado a $19^{\circ} 29^{\prime} \mathrm{N}$ y $98^{\circ} 53^{\prime} \mathrm{O}$, a 2,240 msnm. El clima es templado subhúmedo, con precipitación media anual de $636 \mathrm{~mm}$ y régimen de lluvias en verano con temperatura media anual de $15.2{ }^{\circ} \mathrm{C}^{(14)}$. El suelo es Typic ustipsamments franco arenoso, con pH 7 a 8 , y $2.4 \%$ de materia orgánica(15).

Las praderas se establecieron en febrero de 2010; las gramíneas se sembraron en hileras a 30 $\mathrm{cm}$, mientras que la leguminosa se sembró en forma perpendicular con una distancia de $30 \mathrm{~cm}$; la densidad de siembra fue diferente en cada asociación, dependiendo del porcentaje de cada especie, tomando como base para el pasto ovillo, ballico perenne y trébol blanco en monocultivo a 20, 30 y $5 \mathrm{~kg} \mathrm{ha}^{-1}$, respectivamente. Las praderas no se fertilizaron, y en las estaciones con poca o nula precipitación se proporcionaron riegos a capacidad de campo cada dos semanas. Antes de iniciar la investigación, se realizó una cosecha de homogenización del forraje, mediante un pastoreo con ovinos, cosechando aproximadamente a $5 \mathrm{~cm}$ sobre el nivel del suelo; para su mejor manejo las parcelas estuvieron delimitadas mediante un cerco eléctrico. Posteriormente, los pastoreos se realizaron cada 4 semanas en primavera-verano y cada 5 y 6 semanas durante otoño e invierno, respectivamente, de acuerdo a lo recomendado por otros autores al evaluar praderas de ovillo y ballico perenne ${ }^{(16,17)}$.

Los tratamientos consistieron de las siguientes asociaciones: 20-70-10, 70-20-10, 40-40-20\% de pasto ovillo $(\mathrm{Ov})$, ballico perenne $(\mathrm{Ba})$ y trébol blanco ( $\mathrm{Tr})$, respectivamente y el pasto ovillo en monocultivo (100-00-00), Los cuatro tratamientos se distribuyeron en 12 parcelas experimentales de 9 $x 8 \mathrm{~m}$ en un arreglo de bloques al azar con tres repeticiones. 


\section{Dinámica poblacional de tallos}

Para determinar los cambios en la densidad de tallos, así como sus tasas de aparición y muerte, al inicio del experimento, en cada unidad experimental se colocaron dos aros de PVC de $10.4 \mathrm{~cm}$ de diámetro, los cuales delimitaban un macollo; cuando las praderas eran integradas por la asociación de las dos especies de pastos, cada aro registraba una especie. Todos los tallos presentes dentro del aro se marcaron con anillos de cable de un mismo color, que se consideraron como población inicial. Posteriormente, cada mes, durante dos años, los tallos nuevos se marcaron con anillos de color diferente; se usó un color diferente para cada generación, y los tallos muertos se contaron y se les retiró el anillo.

Los valores población, tasa de aparición y muerte de tallos se multiplicaron por el número de plantas por metro cuadrado; éstas se contaron un día después de cada pastoreo en un cuadro fijo de 1 $\mathrm{m}^{2}$, establecido al azar en cada parcela y repetición, las cuales están reportadas en una primera fase de investigación ${ }^{(18)}$. Con los datos anteriores se estimó: densidad poblacional de tallos (DPT; tallos $\mathrm{m}^{-2}$ ), tasas de aparición (TAT) y muerte (TMT), utilizando las siguientes fórmulas ${ }^{(4)}$ :

$\mathrm{TAT}=\frac{\text { No. de tallos nuevos }}{(\mathrm{DPT} \text { del muestreo anterior) }(\mathrm{T} 2-\mathrm{T} 1)}(100)$
$\mathrm{TMT}=\frac{\text { No. de tallos muertos }}{(\mathrm{DPT} \text { del muestreo anterior) }(\mathrm{T} 2-\mathrm{T} 1)}(100)$

La tasa de sobrevivencia de tallos (TST) se obtuvo de manera indirecta mediante la ecuación: $\mathrm{TST}=100-\mathrm{TMT}$

\section{Peso por tallo}

Un día antes de cada pastoreo, se cosecharon a ras de suelo 10 tallos de pasto ovillo y 10 de ballico perenne, se secaron en una estufa de aire forzado a $55^{\circ} \mathrm{C}$, hasta que alcanzaron un peso constante, y posteriormente se registró su peso. El peso promedio de tallo se obtuvo al dividir el peso de la muestra de tallos entre el número de tallos.

\section{Datos climáticos}

En el periodo experimental la temperatura máxima promedio osciló entre 21 y $26^{\circ} \mathrm{C}$, la mínima promedio mensual entre 3 y $10{ }^{\circ} \mathrm{C}$. La temperatura máxima se presentó en primavera de ambos años, con un promedio de $26^{\circ} \mathrm{C}$; la mínima ocurrió en otoño e invierno con 5.7 y 3.6 ${ }^{\circ} \mathrm{C}$, respectivamente. La precipitación acumulada en el primer año fue de $424.81 \mathrm{~mm}$, en primaveraverano de 2013 se presentó la mayor precipitación con $378.28 \mathrm{~mm}$ (89\%). La precipitación acumulada del segundo año fue de $332.8 \mathrm{~mm}$, obteniendo la mayor precipitación en primavera y verano de 2014 con $289.78 \mathrm{~mm}$ (87 \%) (Figura 1).

Figura 1. Temperaturas media por estación, máxima, mínima, precipitación acumulada y riegos a capacidad de campo durante el periodo de estudio (http://www.cm.colpos.mx/ meteoro/)

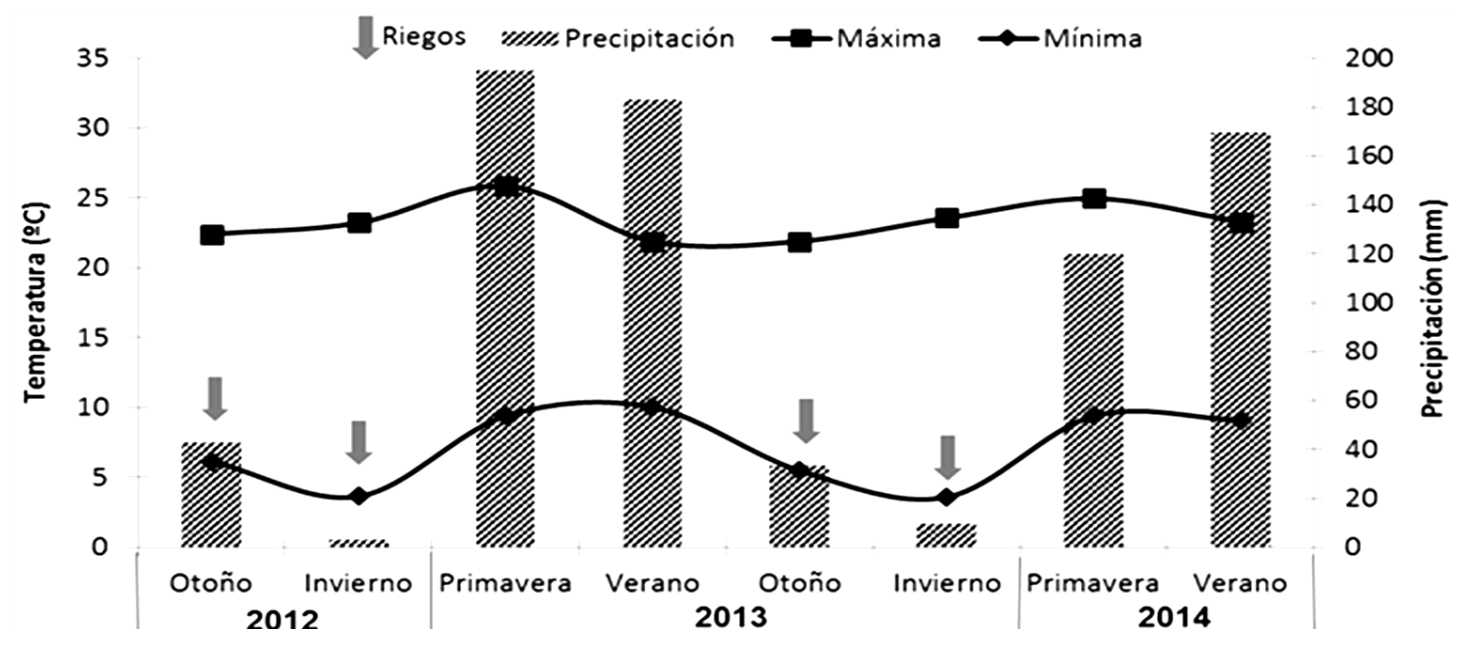




\section{Análisis estadístico}

Los datos obtenidos en las variables de los cuatro tratamientos se organizaron de forma estacional y anual para su análisis de varianza, conforme un diseño de bloques al azar con tres repeticiones, mediante el procedimiento PROC MIXED del programa Statistical Analysis System ${ }^{(19)}$; la comparación de medias se estimó por LSMEANS mediante la prueba " $\mathrm{t}$ " de "Student" a una significancia ajustada $(P<0.05)$.

En pasto ovillo se encontraron diferencias $(P<0.05)$ en las tasa de aparición de tallos debido a efecto de las estaciones del año, presentándose la mayor tasa de aparición en las estaciones de otoño, invierno y primavera del primer año, con un promedio de 1.01 tallos 100 tallos $^{-1} \mathrm{~d}^{-1}$, mientras que la menor se presentó en el verano del segundo año con 0.21 tallos. También se registraron diferencias
$(P<0.05)$ entre años, en el primer año fue de 0.95 y el segundo año 0.47 tallos (Cuadro 1 ).

La tasa de muerte de tallos fue disminuyendo conforme se incrementó la edad de la pradera. En el primer año fue mayor $(P<0.05)$ comparada con el segundo año (0.71 y 0.56 tallos 100 tallos $^{-1} \mathrm{~d}^{-1}$, respectivamente). Entre estaciones también existieron diferencias estadísticas, teniendo en las cuatro estaciones del primer año y otoño del segundo año en donde hubo mayor tasa de muerte de tallos, en promedio 0.71 , y la menor en verano del segundo año con un promedio de 0.36 tallos. La tasa de sobrevivencia de ovillo fue diferente, conforme pasó el tiempo fue aumentando, teniendo en promedio del primer año la menor tasa y el segundo año la mayor con 99.3 y 99.4 tallos, respectivamente $(P<0.05)$. Todas las asociaciones obtuvieron la mayor tasa de sobrevivencia en el primer año con 99.3 tallos, mientras que en el

Cuadro 1. Tasa de aparición, muerte y sobrevivencia de tallos de ovillo (Ov) solo y asociado con ballico perenne (Ba) y trébol blanco $(\mathrm{Tr})$

\begin{tabular}{|c|c|c|c|c|c|c|c|c|c|c|}
\hline \multirow{2}{*}{$\begin{array}{l}\text { Asociación } \\
\text { Ov-Ba-Tr }\end{array}$} & \multicolumn{2}{|c|}{2012} & \multicolumn{4}{|c|}{2013} & \multicolumn{2}{|c|}{2014} & \multicolumn{2}{|c|}{ Promedio } \\
\hline & Otoño & Invierno & Primav. & Verano & Otoño & Invierno & Primav. & Verano & Año 1 & Año 2 \\
\hline \multicolumn{11}{|c|}{ Tasa de aparición de tallos (tallos 100 tallos $^{-1} \mathrm{~d}^{-1}$ ) } \\
\hline $20-70-10$ & $1.26 \mathrm{a}$ & $1.50 \mathrm{a}$ & $0.72^{b}$ & $0.71^{b}$ & $0.60^{c}$ & $0.11^{d}$ & $0.16 \mathrm{bc}$ & $0.14 \mathrm{bc}$ & $1.00 \mathrm{~A}$ & $0.25^{C}$ \\
\hline $70-20-10$ & $1.25 \mathrm{a}$ & $1.08^{a}$ & $1.13^{a}$ & $0.85^{b}$ & $0.95 \mathrm{~b}$ & $0.61^{c}$ & $0.24 d$ & $0.08 \mathrm{e}$ & $1.10 \mathrm{~A}$ & $0.47^{\mathrm{B}}$ \\
\hline $100-00-0$ & $0.87^{a}$ & $1.14^{\mathrm{a}}$ & $1.06 \mathrm{a}$ & $0.95^{a}$ & $0.50^{b}$ & $0.58 \mathrm{~b}$ & $0.51^{b}$ & $0.21^{c}$ & $1.00 \mathrm{~A}$ & $0.45^{B}$ \\
\hline $40-40-20$ & $0.61^{b}$ & $0.83^{a}$ & $0.73^{b}$ & $0.65^{b}$ & $0.95^{a}$ & 1.02 a & $0.48^{c}$ & $0.44^{c}$ & $0.70^{B}$ & $0.72 \mathrm{~A}$ \\
\hline Promedio & 0.99 a & $1.13^{\mathrm{a}}$ & $0.91 \mathrm{a}$ & $0.79 \mathrm{~b}$ & $0.75^{b}$ & $0.58^{c}$ & $0.34 \mathrm{~d}$ & $0.21 \mathrm{e}$ & $0.95^{\mathrm{a}}$ & $0.47^{b}$ \\
\hline \multicolumn{11}{|c|}{ Tasa de muerte de tallos (tallos 100 tallos $^{-1} \mathrm{~d}^{-1}$ ) } \\
\hline $20-70-10$ & $0.52 \mathrm{~b}$ & $0.76^{a}$ & $0.85 \mathrm{a}$ & $0.73^{a}$ & $0.62 \mathrm{~b}$ & $0.47 \mathrm{c}$ & $0.48^{c}$ & $0.23^{d}$ & $0.71 \mathrm{~A}$ & $0.45^{\mathrm{B}}$ \\
\hline $70-20-10$ & $0.71^{b}$ & $0.64^{b}$ & $0.76^{a}$ & $0.82^{a}$ & $0.77^{a}$ & 0.68 ab & $0.53 b c$ & $0.42^{c}$ & $0.73 \mathrm{~A}$ & $0.60 \mathrm{~A}$ \\
\hline $100-00-0$ & $0.83^{a}$ & $0.64 \mathrm{~b}$ & $0.73^{b}$ & $0.82^{a}$ & $0.79 a$ & $0.6^{b}$ & $0.70^{b}$ & $0.36^{c}$ & $0.75^{\mathrm{A}}$ & $0.61 \mathrm{~A}$ \\
\hline $40-40-20$ & $0.83^{a}$ & $0.52^{c}$ & $0.54^{c}$ & $0.74^{a}$ & $0.63^{b}$ & $0.65^{b}$ & $0.62^{b}$ & $0.46^{d}$ & $0.65^{B}$ & $0.59 \mathrm{~A}$ \\
\hline Promedio & 0.72 a & 0.64 a & 0.72 a & $0.77^{a}$ & 0.70 a & $0.6^{b}$ & $0.58 \mathrm{~b}$ & $0.36 c$ & 0.71 a & $0.56 \mathrm{~b}$ \\
\hline \multicolumn{11}{|c|}{ Tasa de sobrevivencia de tallos (tallos 100 tallos $^{-1} \mathrm{~d}^{-1}$ ) } \\
\hline $20-70-10$ & $99.5^{\mathrm{a}}$ & $99.2^{b}$ & $99.2^{b}$ & $99.3^{b}$ & $99.4 \mathrm{ab}$ & $99.5^{\text {a }}$ & $99.5^{\mathrm{a}}$ & $99.8^{a}$ & $99.3^{\mathrm{A}}$ & $99.6 \mathrm{~A}$ \\
\hline $70-20-10$ & $99.3^{b}$ & $99.4 \mathrm{ab}$ & $99.2^{b}$ & $99.2^{b}$ & $99.2^{b}$ & $99.3^{b}$ & $99.5^{\mathrm{a}}$ & 99.6 a & $99.3^{A}$ & $99.4^{B}$ \\
\hline $100-00-0$ & $99.2^{b}$ & 99.4 a & 99.3 ab & $99.2^{b}$ & $99.2^{b}$ & 99.4 a & $99.3 \mathrm{ab}$ & 99.6 a & 99.2 B & $99.4^{B}$ \\
\hline $40-40-20$ & $99.2^{b}$ & $99.5^{a}$ & $99.5^{a}$ & $99.3^{b}$ & $99.4 \mathrm{ab}$ & 99.4 ab & $99.4 \mathrm{ab}$ & $99.5^{\text {a }}$ & 99.3 A & $99.4^{B}$ \\
\hline Promedio & $99.3^{b}$ & $99.4 \mathrm{ab}$ & $99.3^{b}$ & $99.2^{b}$ & $99.3^{b}$ & 99.4 ab & $99.4 \mathrm{ab}$ & 99.6 a & $99.3^{b}$ & 99.4 a \\
\hline
\end{tabular}

Primav. $=$ primavera.

$\mathrm{abc}=$ Medias con la misma literal minúscula en una misma hilera, no son diferentes $(P<0.05)$.

$\mathrm{ABC}=$ Medias con la misma literal mayúscula en una misma columna, no son diferentes $(P<0.05)$. 
segundo año la asociación 20-70-10 de Ov-Ba-Tr obtuvo la mayor tasa de sobrevivencia con 99.6 tallos

Varios investigadores reportan en tasa de aparición de tallos para pasto ovillo diferencias entre asociaciones durante el invierno, y presentando dos picos más altos en primavera y otoño ${ }^{(11,20)}$. La velocidad de crecimiento de las plantas forrajeras depende de los factores ambientales, particularmente el clima, por lo que las variaciones observadas en tasa de aparición y muerte de tallos, podría deberse a los cambios drásticos en la calidad de la luz, la temperatura óptima de crecimiento de la especie, y a la disminución progresiva en la biomasa verde conforme crece la pradera ${ }^{(8)}$. Esto ocasiona que la tasa de formación de tallos se reduzca como resultado de una respuesta fotomorfogénica y el sombreado de las capas inferiores de la pradera. Por otro lado, cuando varias especies se encuentran en su máximo potencial de crecimiento, el componente más importante del rendimiento de forraje es el peso por tallo(21), por lo que la alta tasa de muerte de tallos registrada en el primer año de evaluación, se debió a la mayor tasa de crecimiento individual de cada tallo, lo que sombreó a los tallos pequeños, presentes en los estratos inferiores de la pradera, incrementando la mortalidad de los mismos ${ }^{(1)}$.

En la tasa de aparición, muerte y sobrevivencia de tallos de ballico perenne se encontraron diferencias $(P<0.05)$. La tasa de aparición de tallos del primer año fue menor al segundo año, $0.66 \mathrm{y}$ 0.84 tallos 100 tallos $^{-1} \mathrm{~d}^{-1}$, respectivamente. En la asociación 20-70-10 de Ov-Ba-Tr tendió a aumentar la tasa de aparición de tallos, en promedio fue de 0.61 y 1.19 tallos para el primer y segundo año, respectivamente. Las asociaciones 70-20-10 y $40-$ 40-20 de Ov-Ba-Tr fueron caso contrario, ya que la tasa de aparición de tallos tendió a disminuir conforme transcurrió el tiempo (Cuadro 2). Existen reportes en ballico perenne ${ }^{(11)}$ con la mayor tasa de aparición en abril y noviembre (primavera y otoño), similares a lo registrado en esta investigación, ya que en otoño de ambos años, se encontraron las

Cuadro 2. Tasa de aparición, muerte y sobrevivencia de tallos de ballico perenne (Ba) asociado con ovillo (Ov) y trébol blanco (Tr)

\begin{tabular}{|c|c|c|c|c|c|c|c|c|c|c|}
\hline \multirow{2}{*}{$\begin{array}{l}\text { Asociación } \\
\text { Ov-Ba-Tr }\end{array}$} & \multicolumn{2}{|c|}{2012} & \multicolumn{4}{|c|}{2013} & \multicolumn{2}{|c|}{2014} & \multicolumn{2}{|c|}{ Promedio } \\
\hline & Otoño & Invierno & Primav. & Veranc & o Otoño & Invierno & Primav. & Verano & Año 1 & Año 2 \\
\hline \multicolumn{11}{|c|}{ Tasa de aparición de tallos (tallos 100 tallos $^{-1} \mathrm{~d}^{-1}$ ) } \\
\hline $20-70-10$ & $0.75^{b}$ & $0.80 \mathrm{~b}$ & $0.54 c$ & $0.34 \mathrm{c}$ & $1.20 \mathrm{a}$ & 1.02 a & $1.18^{a}$ & 1.35 a & $0.61 \mathrm{~B}$ & $1.19 \mathrm{~A}$ \\
\hline $70-20-10$ & 0.98 a & $0.95^{a}$ & $0.67^{b}$ & $0.59 c$ & $0.84 \mathrm{ab}$ & $0.78^{b}$ & $0.72^{b}$ & $0.76^{b}$ & $0.79 \mathrm{~A}$ & $0.77^{\mathrm{B}}$ \\
\hline $40-40-20$ & $0.92^{a}$ & $0.98^{a}$ & $0.60 \mathrm{~b}$ & $0.56^{b}$ & $0.96^{a}$ & $0.56^{b}$ & $0.37^{c}$ & $0.43 \mathrm{bc}$ & $0.58^{B}$ & $0.58 \mathrm{c}$ \\
\hline Promedio & $0.88 \mathrm{ab}$ & $0.78^{b}$ & $0.60^{c}$ & $0.49 d$ & $1.00 \mathrm{a}$ & $0.78^{b}$ & $0.75^{b}$ & $0.84 \mathrm{ab}$ & $0.66^{b}$ & $0.84{ }^{a}$ \\
\hline \multicolumn{11}{|c|}{ Tasa de muerte de tallos (tallos 100 tallos $^{-1} \mathrm{~d}^{-1}$ ) } \\
\hline $20-70-10$ & $0.56 \mathrm{bc}$ & $0.83^{a}$ & $0.61^{b}$ & $0.44^{c}$ & $0.62^{b}$ & $0.85^{a}$ & $0.77 \mathrm{a}$ & $0.90^{a}$ & $0.61^{\mathrm{B}}$ & $0.78^{A}$ \\
\hline $70-20-10$ & $0.83 a$ & $0.86^{a}$ & $0.75 \mathrm{ab}$ & $0.63^{b}$ & $0.68^{b}$ & $0.74 \mathrm{ab}$ & $0.76 \mathrm{ab}$ & $0.68^{b}$ & $0.76^{A}$ & $0.71 \mathrm{~A}$ \\
\hline $40-40-20$ & $0.82^{a}$ & $0.84^{a}$ & $0.78^{a}$ & $0.62^{b}$ & $0.52^{c}$ & 0.77 a & $0.53^{c}$ & $0.40^{c}$ & $0.76^{A}$ & $0.55^{B}$ \\
\hline Promedio & $0.73 a b$ & $0.84 a$ & $0.71 \mathrm{ab}$ & $0.56^{c}$ & $0.60^{c}$ & $0.78^{a}$ & $0.68^{b}$ & $0.66 \mathrm{bc}$ & $0.71^{a}$ & $0.68^{b}$ \\
\hline \multicolumn{11}{|c|}{ Tasa de sobrevivencia de tallos (tallos 100 tallos $^{-1} \mathrm{~d}^{-1}$ ) } \\
\hline $20-70-10$ & $99.4 \mathrm{ab}$ & $99.2^{b}$ & $99.4 \mathrm{ab}$ & 99.6 a & $99.4 \mathrm{ab}$ & $99.2^{b}$ & $99.2^{b}$ & $99.1^{b}$ & $99.4 \mathrm{~A}$ & $99.2^{B}$ \\
\hline $70-20-10$ & $99.2^{c}$ & $99.1^{c}$ & $99.3^{b}$ & 99.4 a & $99.3^{b}$ & $99.3^{b}$ & $99.2^{c}$ & $99.3^{b}$ & $99.2^{B}$ & $99.4^{\mathrm{A}}$ \\
\hline $40-40-20$ & $99.2 \mathrm{~b}$ & 99.2 b & $99.2 \mathrm{~b}$ & $99.4 a b$ & 99.5 a & $99.2^{b}$ & 99.5 a & 99.6 a & $99.2 \mathrm{~B}$ & $99.4 \mathrm{~A}$ \\
\hline Promedio & $99.3^{b}$ & $99.2^{c}$ & $99.3^{b}$ & 99.4 a & $99.4^{\text {a }}$ & $99.2^{c}$ & $99.3^{b}$ & $99.3^{b}$ & $99.2^{b}$ & $99.3^{\text {a }}$ \\
\hline
\end{tabular}

Primav= primavera.

$\mathrm{abc}=$ Medias con la misma literal minúscula en una misma hilera, no son diferentes $(P<0.05)$.

$\mathrm{ABC}=$ Medias con la misma literal mayúscula en una misma columna, no son diferentes $(P<0.05)$. 
mayores tasas de aparición de tallos. En otra investigación ${ }^{(22)}$ al evaluar ballico perenne a diferentes frecuencias e intensidades de pastoreo, registraron la mayor tasa de aparición de tallos en otoño. En praderas puras de ballico perenne ${ }^{(10)}$, reportaron las mayores tasas de aparición de tallos en verano e invierno, las cuales duplicaron a las de otoño y primavera.

Figura 2. Cambios mensuales en la densidad de tallos de ovillo (Ov) asociado con ballico perenne (Ba) y trébol blanco ( $\mathrm{Tr}$ ) en diferentes proporciones: a) $20 \%$ de ovillo (20-70-10), b) $70 \%$ de ovillo (70-20-10), c) $100 \%$ de ovillo (100-00-00) y d) $40 \%$ de ovillo (40-40-20)

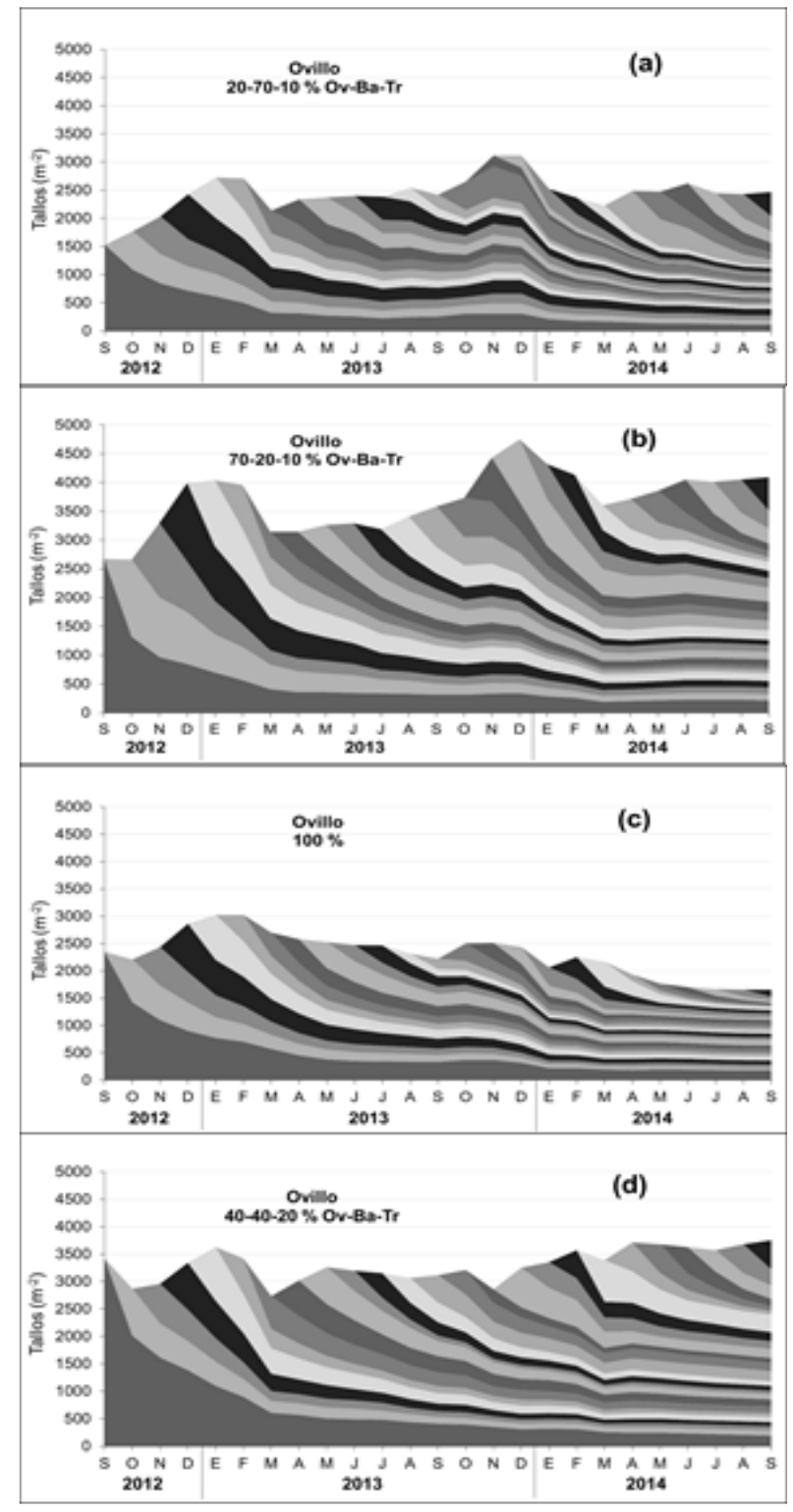

La asociación 20-70-10 de Ov-Ba-Tr registró la menor tasa de muerte de tallos de ballico perenne en el primer año, y no se presentaron diferencias estadísticas entre las otras asociaciones; en el segundo año la menor tasa de muerte de tallos se presentó en la asociación 40-40-20 (Cuadro 2). En promedio la tasa de muerte de tallos fue mayor en el primer año que en el segundo $(P<0.05)$. Durante el primer año de evaluación la tasa de muerte de tallos fue similar entre estaciones; en el segundo año, la tasa de muerte fue mayor en invierno, seguida de primavera, verano, y la menor en otoño.

La tasa de sobrevivencia de tallos de ballico perenne aumentó del primer al segundo año de 99.2 a 99.3 tallos 100 tallos $^{-1} \mathrm{~d}^{-1}(P<0.05)$. En verano del primer año y otoño del segundo se encontró la mayor tasa de sobrevivencia con un promedio de 99.4 tallos y en la estación de invierno de ambos años con un promedio de 99.2 tallos la menor tasa de sobrevivencia.

En otra investigación en asociaciones con ballico perenne, se reporta variación en la tasa de mortalidad de tallos, siendo en octubre cuando se presentó la mayor mortalidad, y lo atribuyen principalmente a la presencia de heladas ${ }^{(11)}$. Otros autores $^{(22)}$ obtuvieron en praderas de ballico perenne en monocultivo, la mayor tasa de muerte en verano (septiembre) y lo atribuyen a la competencia por luz y nutrientes, así como a la defoliación de tallos reproductivos que son la fuente de nutrientes de los tallos jóvenes.

En las Figuras 2 y 3 se muestran la densidad de tallos a lo largo del periodo experimental. De noviembre a marzo se registró la mayor densidad de tallos, independientemente la asociación y año (Figura 2). La asociación 70-20-10 de Ov-Ba-Tr fue la que presentó mayor densidad de tallos de ovillo con un promedio de 3,750 tallos $\mathrm{m}^{-2}$, mientras que la pradera con ovillo en monocultivo (100-00-00) registró la menor densidad de tallos a lo largo de la investigación, con un promedio de 2,400 tallos $\mathrm{m}^{-2}$; además en este tratamiento la densidad de tallos tendió a disminuir conforme aumentó el tiempo.

En promedio todas las asociaciones presentaron menor densidad de tallos de abril a septiembre (primavera- verano), y se le puede atribuir a la 
Figura 3. Cambios mensuales en la densidad de tallos de ballico perenne $(\mathrm{Ba})$ asociado con ovillo $(\mathrm{Ov})$ y trébol blanco $(\mathrm{Tr})$ en diferentes proporciones: a) $70 \%$ de ballico perenne (20-70-10), b) $20 \%$ de ballico perenne (70-20-10) y c) $40 \%$ de ballico perenne (40-40-20)

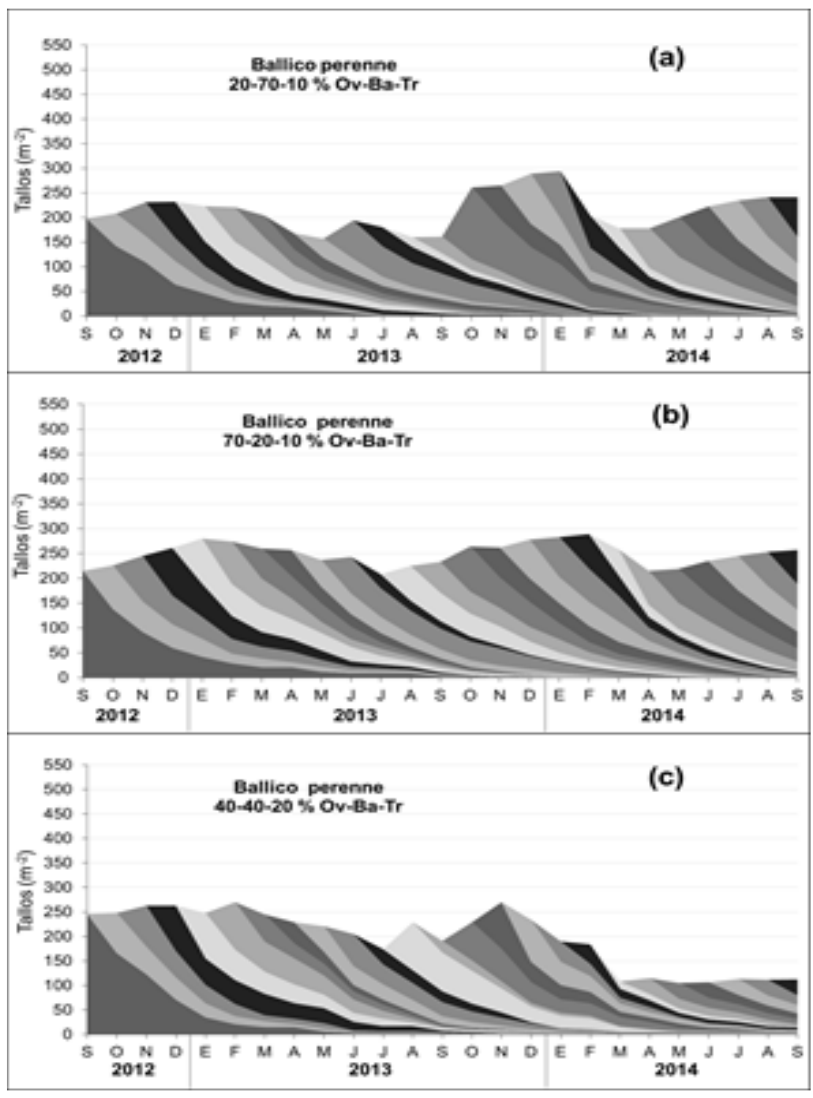

mayor temperatura registrada en esas estaciones, ya que los tallos tienden a tener mayor peso (Cuadro 3) y área foliar $y$, por lo tanto, sombrean a los nuevos tallos, aumentando su mortalidad ${ }^{(5)}$. Otro factor que afectó el comportamiento de los tallos fueron las competencias inter e intra-especifica por nutrientes, agua, luz y espacio(23), ya que las plantas no rebrotan en una pradera como individuos aislados, sino como una población usualmente densa, donde la vegetación que los rodea ejerce una influencia muy fuerte sobre las características inherentes de cada especie, por medio de la competencia ${ }^{(24)}$. Otros autores, en el valle de México, reportan en ovillo asociado con las mismas especies $^{(11)}$ y en monocultivo ${ }^{(12)}$ datos similares a lo encontrado en esta investigación.

En ballico perenne la dinámica poblacional de tallos presentó una gran variación en su comportamiento, dependiendo del porcentaje inicial en la asociación (Figura 3). Al igual que en el pasto ovillo, todas las asociaciones presentaron la mayor densidad poblacional de tallos en los meses con la temperatura mínima (otoño - invierno). La asociación 70-20-10 de Ov-Ba-Tr tuvo la mayor densidad de tallos de ballico perenne con un promedio de 260 tallos $\mathrm{m}^{-2}$, mientras que en la asociación 40-40-20 de Ov-Ba-Tr se registró la menor densidad, en promedio 190 tallos $\mathrm{m}^{-2}$, y se presentó una marcada disminución en los últimos

Cuadro 3. Peso de tallos de ovillo $(\mathrm{Ov})$ y ballico perenne $(\mathrm{Ba})$ asociados con trébol blanco $(\mathrm{Tr})$

\begin{tabular}{|c|c|c|c|c|c|c|c|c|c|c|}
\hline \multirow{2}{*}{$\begin{array}{l}\text { Asociación } \\
\text { Ov-Ba-Tr }\end{array}$} & \multicolumn{2}{|r|}{2012} & \multicolumn{4}{|c|}{2013} & \multicolumn{2}{|c|}{2014} & \multicolumn{2}{|c|}{ Promedio } \\
\hline & Otoño & Invierno & Primav. & Verano & Otoño & Invierno & Primav. & Verano & Año 1 & Año 2 \\
\hline \multicolumn{11}{|c|}{ Pasto ovillo (g tallo-1) } \\
\hline $20-70-10$ & $0.19^{c}$ & $0.14^{c}$ & $0.35^{a}$ & $0.23^{b}$ & $0.18^{c}$ & $0.13^{c}$ & $0.33^{a}$ & $0.23^{b}$ & $0.23 \mathrm{~A}$ & $0.21 \mathrm{~A}$ \\
\hline $70-20-10$ & $0.28 a$ & $0.18^{b}$ & $0.39 a$ & $0.20 \mathrm{~b}$ & $0.25 a b$ & $0.18^{b}$ & $0.34 \mathrm{a}$ & $0.22 \mathrm{~b}$ & $0.26 \mathrm{~A}$ & $0.24 \mathrm{~A}$ \\
\hline $100-00-0$ & $0.13^{a}$ & $0.09 \mathrm{a}$ & 0.14 a & $0.12^{a}$ & $0.13^{a}$ & 0.09 a & $0.13^{a}$ & $0.12^{a}$ & $0.12^{B}$ & $0.11^{\mathrm{B}}$ \\
\hline $40-40-20$ & $0.22 \mathrm{~b}$ & $0.12^{c}$ & 0.39 a & $0.30 \mathrm{ab}$ & $0.13^{c}$ & $0.12^{c}$ & 0.34 a & $0.31 \mathrm{ab}$ & $0.25^{A}$ & $0.22 \mathrm{~A}$ \\
\hline Promedio & $0.20^{b}$ & $0.13^{c}$ & $0.31^{a}$ & $0.21^{b}$ & $0.17 \mathrm{bc}$ & $0.13^{c}$ & $0.28^{a}$ & $0.22 \mathrm{~b}$ & $0.21 \mathrm{a}$ & $0.19 b$ \\
\hline \multicolumn{11}{|c|}{ Ballico perenne ( $\mathrm{g}$ tallo-1) } \\
\hline $20-70-10$ & $0.20 \mathrm{bc}$ & $0.16^{c}$ & $0.43 a$ & $0.21 \mathrm{~b}$ & $0.19 b c$ & $0.16^{c}$ & $0.41^{a}$ & $0.22 \mathrm{bc}$ & 0.25 & $0.24 \mathrm{~A}$ \\
\hline $70-20-10$ & $0.23^{b}$ & $0.16^{c}$ & $0.44 a$ & $0.22 b$ & $0.20 \mathrm{bc}$ & $0.16^{c}$ & $0.42^{a}$ & $0.22 \mathrm{~b}$ & 0.25 & $0.25^{A}$ \\
\hline $40-40-20$ & $0.14^{c}$ & $0.12^{c}$ & $0.42^{a}$ & $0.28^{b}$ & $0.11^{\mathrm{c}}$ & $0.09 \mathrm{c}$ & $0.41^{a}$ & $0.24^{b}$ & 0.24 & $0.21^{c}$ \\
\hline Promedio & $0.19 b c$ & $0.14^{c}$ & $0.43 a$ & $0.23 \mathrm{~b}$ & $0.16^{c}$ & $0.13^{c}$ & $0.41 \mathrm{a}$ & $0.22^{b}$ & 0.24 & 0.23 \\
\hline
\end{tabular}

Primav= primavera.

$a b c=$ Medias con la misma literal minúscula en una misma hilera, no son diferentes $(P<0.05)$. 
meses del segundo año de evaluación. La poca densidad de tallos que presentó esta especie se puede atribuir a la edad de la pradera, ya que al inicio de la investigación llevaba tres años desde la siembra ${ }^{(18)}$. Otro factor que pudo afectar la disminución en densidad de tallos fue la temperatura promedio máxima registrada durante primavera (26 ${ }^{\circ} \mathrm{C}$; Figura 1), la cual superó considerablemente a la temperatura óptima de crecimiento de ballico perenne que es de $18{ }^{\circ} \mathrm{C}^{(25)}$. Al respecto, algunos autores $^{(4,26)}$ han consignado que las altas temperaturas ocasionan reducción en el crecimiento y tasa de acumulación de forraje, por influencia directa de una menor tasa de aparición y expansión foliar ${ }^{(20,27)}$. Varios investigadores reportan en ballico perenne mayor densidad de tallos en los meses con la temperatura mínima(11,28).

El peso por tallo de pasto ovillo solo y asociado con ballico perenne y trébol blanco fue mayor $(P<0.05)$ en el primer año de evaluación $(0.21 \mathrm{vs}$ $0.19 \mathrm{~g} \mathrm{tallo}^{-1}$ ) en comparación con el segundo año (Cuadro 3). En primavera e invierno de ambos años se encontró el mayor y menor peso por tallo de pasto ovillo con promedios de 0.29 y $0.13 \mathrm{~g}$ tallo $^{-1}$ $(P<0.05)$. En las praderas de pasto ovillo en monocultivo (100-00-00) se presentó el menor peso por tallo $\left(0.11 \mathrm{~g} \mathrm{tallo}^{-1}\right)$, mientras que en las asociaciones en promedio fue de $0.23 \mathrm{~g}^{\text {tallo }}{ }^{-1}$ $(P<0.05)$.

El mayor peso por tallo en las asociaciones se le puede atribuir a que existe un mayor aprovechamiento de recursos, ya que al estar asociado con la leguminosa y al poseer bacterias del género Rhizobium, fijan nitrógeno atmosférico al suelo y mediante simbiosis es aprovechado por las gramíneas, lo que ocasiona mayor rendimiento, peso por tallo y calidad, en comparación con la gramínea en monocultivo ${ }^{(29)}$. Estos resultados son similares a los encontrados por diferentes investigadores, quienes evaluaron el pasto ovillo asociado con ballico perenne y trébol blanco(30-33) y en monocultivo ${ }^{(12,14,17)}$, obteniendo en primavera $y$ verano el mayor forraje producido, lo cual está estrechamente correlacionado con el mayor peso por tallo. En asociaciones se han registrado mayores pesos por tallo de pasto ovillo en verano, relacionándolo con la mayor temperatura, similar a lo encontrado en esta investigación ${ }^{(11)}$.

El ballico perenne no presentó diferencias estadísticas en el peso por tallo en promedio por año, y tampoco entre asociaciones en el primer año. Sin embargo, sí registró diferencias (Cuadro 3) entre asociaciones en el segundo año de evaluación siendo 20-70-10 y 70-20-10 de Ov-Ba-Tr, donde se registró el mayor peso por tallo con un promedio de $0.24 \mathrm{~g}$ tallo ${ }^{-1}$, mientras que el menor peso lo obtuvo la asociación 40-40-20 de Ov-Ba-Tr con $0.21 \mathrm{~g}$ $(P<0.05)$. En primavera de ambos años se presentó el mayor peso por tallo de ballico perenne con un promedio de $0.42 \mathrm{~g}$, mientras que el menor peso lo presentan principalmente las estaciones de invierno y otoño de ambos años con un promedio de $0.15 \mathrm{~g}$ $(P<0.05)$.

El mayor y menor peso por tallo de ballico perenne al igual que en pasto ovillo es variable dependiendo de la asociación, temperatura máxima y mínima, mayor y menor horas luz registradas en el tiempo que transcurrió la investigación (Figura 1). Otros autores ${ }^{(22)}$ en ballico perenne mencionan un mayor peso por tallo que en esta investigación, con 2.2 y $1.8 \mathrm{~g} \mathrm{tallo}^{-1}$ en verano e invierno, respectivamente; sin embargo, era el primer año de evaluación, y en esta investigación llevaba tres años desde la siembra, por lo tanto la persistencia de ballico perenne disminuyó notablemente ${ }^{(18)}$ y por consecuencia el peso por tallo. Otros autores, trabajando en praderas de ballico perenne en monocultivo(10) y asociado con ovillo y trébol blanco $^{(11)}$ reportaron durante primavera- verano mayor peso por tallo y en otoño e invierno el menor, similar a lo encontrado en esta investigación.

La tasa de aparición y muerte de tallos en ambas gramíneas tendieron a disminuir; en contraste, la tasa de sobrevivencia aumentó conforme pasó el tiempo. La densidad poblacional de tallos de ballico perenne fue menor que la población de tallos de ovillo, y fue disminuyendo conforme aumentó la edad de la pradera. El mayor y menor peso por tallo en las dos gramíneas se presentó durante las estaciones de primavera e invierno; sin embargo, también en estas estaciones se observó menor y mayor densidad de tallos, 
dando como resultado una compensación tamaño/densidad. Se recomienda utilizar las asociaciones 70-20-10 y 40-40-20 de Ov-Ba-Tr, ya que obtuvieron mayor peso y densidad de tallos de ovillo.

\section{LITERATURA CITADA}

1. Matthew $C$. Seasonal patterns of rood, tiller and leaf production in a Grassland Ruanui ryegrass sward. Proc N Z Grass Assoc 1996;(58):73-76.

2. Matthew C, Lemaire G, Sackville-Hamilton NR, Hernández-Garay A. A modified selfthinning equation to describe size/density relationships for defoliated swards. Annals Botany 1995;76(6):579587.

3. Hodgson J. Grazing management. Science into practice. Harlow, England: Longman Scientific and Technical; 1990.

4. Hernández-Garay A, Hodgson J, Matthew C. Effect of spring grazing management on perennial ryegrass/White clover pastures. 2. Tiller and growing point densities and population dynamics. N Z J Agr Res 1997;40(1):37-50.

5. Hernández-Garay A, Matthew C, Hodgson J. Tiller size/density compensation in perennial ryegrass miniature swards subject to differing defoliation heights and a proposed productivity index. Grass Forage Sci 1999;54(4):347-356.

6. Lemaire, G. Ecophysiology of grasslands: Dynamic aspects of forage plant population in swards. In: Proc XVII Int Grass Cong. Brazilian Soc Anim Husb Sociedade Brasileira de Zootecnia. Sao Paulo, Brasil. 2001:29-37.

7. Pérez BMT, Hernández-Garay A, Pérez PJ, Herrera HJG, Bárcena GR. Respuesta productiva y dinámica de rebrote del ballico perenne a diferentes alturas de corte. Téc Pecu Méx 2002;40(3):251-263.

8. Matthew C, Val Loo ER, Tom ER, Dawson LA, Care DA. Understanding shoot and root development. Proc XIX Int Grass Cong. Sao Paulo, Brasil. 2001.

9. Duchini PG, Guzatti GC, Ribeiro Filho HMN, Sbrissia AF. Tiller size/density compensation in temperate climate grasses grown in monoculture or in intercropping systems under intermittent grazing. Grass Forage Sci 2013;(69):655-665.

10. Velasco ZME, Hernández-Garay A, González HVA. Cambios en componentes del rendimiento de una pradera de ballico perenne, en respuesta a la frecuencia de corte. Rev Fitotecnia Mex 2007;30(1):79-87.

11. Castro RR, Hernández-Garay A, Ramírez RO, Aguilar BG, Enríquez QJF, Mendoza PSI. Crecimiento en longitud foliar y dinámica de población de tallos de cinco asociaciones de gramíneas y leguminosa bajo pastoreo. Rev Mex Cienc Pecu 2013;4(2):201-215.

12. Hernández GFJ, Hernández-Garay A, Ortega JE, Enríquez QJF, Velázquez MM. Comportamiento productivo del pasto ovillo (Dactylis glomerata L.) en respuesta al pastoreo. Agronomía Mesoamericana 2015;26(1):33-42.

13. Hodgson J, Da Silva SC. Options in tropical pasture management. Proc Ann Meet Brazilian Soc Anim Sci. Recife, Brazil. 2002:180202.
14. García E. Modificaciones al sistema de clasificación climática de Koppen. $4^{a}$ ed. Ciudad de México: Universidad Nacional Autónoma de México; 2004.

15. Ortíz SC. Colección de monolitos. Depto. Génesis de suelos. Edafología, IRENAT. Colegio de Postgraduados. Montecillo, Texcoco, Estado de México. 1997.

16. Velasco ZME, Hernández-Garay A, González HVA. Curvas estacionales de crecimiento del ballico perenne. Rev Fitotecnia Mex 2002;(25):97-106.

17. Velasco ZME, Hernández-Garay A, González HVA, Pérez PJ, Vaquera HH, Galvis SA. Curva de crecimiento y acumulación estacional del pasto ovillo (Dactylis glomerata L.). Téc Pecu Méx 2001;39(1):1-14.

18. Rojas GAR, Hernández-Garay A, Quero CAR, Guerrero RJD, Ayala W, Zaragoza RJL, Trejo LC. Persistencia de Dactylis glomerata L. solo y asociado con Lolium perenne L. y Trifolium repens $\mathrm{L}$. Rev Mex Cienc Agr 2016;7(4):885-895.

19. SAS. Institute. SAS/STAT $\AA$ 9.2. User's Guide Release. Cary, NC: SAS Institute Inc. USA. 2009.

20. Durand JL, Schaufele R, Gastal F. Grass leaf elongation rate as a function of developmental stage and temperature: Morphological analysis and modeling. Annals Botany 1999;83(5):577-588.

21. Hernández-Garay $\mathrm{A}$, Matthew $\mathrm{C}$, Hodgson $\mathrm{J}$. The influence of defoliation height on dry-matter partitioning and $\mathrm{CO}_{2}$ exchange of perennial ryegrass miniature swards. Grass Forage Sci 2000;55(4):372-376.

22. Garduño VS, Pérez PJ, Hernández-Garay A, Herrera HJG, Martínez HPA, Joaquín TBM. Rendimiento y dinámica de crecimiento estacional de ballico perenne, pastoreado con ovinos a diferentes frecuencias e intensidades. Téc Pecu Méx 2009;47(2):189-202.

23. Chapman DF, Lemaire G. Morphogenetic and structural determinants of plant regrowth after defoliation. Proc XVII Int Grassland Cong. New Zealand and Australia 1993:95-104.

24. Sanderson MA, Elwinger GF. Plant density and environment effects on orchardgrass-white clover mixtures. Crop Sci 2002;42(6):20552063.

25. Brock JL, Tilbrook JC. Effect of cultivar of white clover on plant morphology during the establishment of mixed pastures under sheep grazing. N Z J Agr Res 2000;43(3):335-343.

26. Duru M, Ducrocq H. Growth and senescence of the successive grass leaves on a tiller. Ontogenic development and effect of temperature. Annals Botany 2000;85(5):635-643.

27. Horrocks R, Vallentine JF. Harvested forages. Oval Road, London, USA: Academic Press; 1999.

28. Ganderats FS, Hepp KC. Mecanismos de crecimiento de Lolium perenne, Festuca arundinacea y Dactylis glomerata en la zona intermedia de Aysén. Agr Téc 2003;63(2):259-265.

29. Nyfeler D, Huguenin-Elie O, Suter M, Frossard E, Lüscher A. Grasslegume mixtures can yield more nitrogen than legume pure stands due to mutual stimulation of nitrogen uptake from symbiotic and non-symbiotic sources. Agr Ecosys Environ 2011;140:155-163.

30. Castro RR, Hernández-Garay $A$, Vaquera HH, Hernández PGJ, Quero CAR, Enríquez QJF, Martínez HPA. Comportamiento productivo de asociaciones de gramíneas con leguminosas en pastoreo. Rev Fitotecnia Mex 2012;35(1):87-95. 
31. Moreno CMA, Hernández-Garay A, Vaquera HH, Trejo LC, Escalante EJA, Zaragoza RJL, Joaquín TBM. Productividad de siete asociaciones y dos praderas puras de gramíneas y leguminosas en condiciones de pastoreo. Rev Fitotec Mex 2015;38(1):101108.

32. Flores SEJ, Hernández-Garay A, Guerrero RJD, Quero CAR, Martínez HPA. Productividad de asociaciones de pasto ovillo (Dactylis glomerata L.), ballico perenne (Lolium perenne L.) y trébol blanco (Trifolium repens L.). Rev Mex Cienc Pecu 2015;6(3):337347.

33. Rojas GAR, Hernández-Garay A, Ayala W, Mendoza PSI, Joaquín SC, Vaquera HH, Santiago HMA. Comportamiento productivo con praderas con distintas combinaciones de ovillo (Dactylis glomerata L.), ballico perenne (Lolium perenne L.) y trébol blanco (Trifolium repens L.). Rev Fac Cienc Agr. Universidad Nacional de Cuyo. Mendoza. Argentina. 2016 [en prensa]. 